\title{
Z-contrast imaging for elemental analysis: Single atoms to clusters
}

\author{
M. Cem Akatay, Wharton Sinkler, Sergio I. Sanchez, Steven A. Bradley
}

UOP LLC, A Honeywell Company

With the advance of aberration corrected scanning transmission electron microscopy (AC-STEM), electron beam can be focused on a fine point with a flux high enough to create significant excitation events that can be detected by the ever improving energy dispersive X-ray detectors or electron energy loss spectrometers enabling single atom elemental analysis. Tizei et al. and Suenage et al. among many others have shown that element specific chemical signature from single atoms could be obtained by means of EDX and EELS. Such examples are not always relevant for practical applications since they utilize an extremely low intensity background support such as carbon nanotubes/films or trap the atoms within well-defined carbon nanostructures to minimize mobility under the beam $[1,2]$.

Another approach for element specific analysis is to utilize Z-contrast inherent to the high angle annular dark field STEM (HAADF-STEM), where the intensity can be related to atomic number, Z. Krivanek et al. were able to differentiate between $\mathrm{B}, \mathrm{N}$ and $\mathrm{O}$ on graphene using that relationship [3]. Their study benefitted greatly from uniform thickness of graphene sheets which also has low background intensity making the subsequent differentiation among elements with higher accuracy.

This work focuses on employing the Z-contrast imaging method on not-so-ideal structures to differentiate elements from other possible elements within the structure by quantifying the HAADF intensity without any need for detector calibration. Pt-Pd catalyst supported on $\mathrm{Al}_{2} \mathrm{O}_{3}$ is selected as a challenging structure to demonstrate this capability due to porous oxide structure resulting in nonuniform thickness with significant HAADF intensity background. The data is collected on an aberrationcorrected sub-Ångstrom resolution scanning transmission electron microscope. Pt atoms $(Z=78)$ are 1.7 times heavier than $\mathrm{Pd}$ atoms $(\mathrm{Z}=46)$ resulting in decent contrast difference to be utilized by HAADFSTEM imaging. Monometallic Pt on $\mathrm{Al}_{2} \mathrm{O}_{3}$ sample was analyzed as a reference to establish the study on the bimetallic $\mathrm{Pt} / \mathrm{Pd}$ on $\mathrm{Al}_{2} \mathrm{O}_{3}$ sample.

HAADF-STEM micrographs on both samples were collected. HAADF-intensity of individual atoms were measured from the micrographs and intensity histogram is plotted as shown in Figure 1. The bimetallic sample exhibited higher standard deviation than the monometallic sample indicative of its bimetallic nature owing to Pt and Pd single atoms.

The findings on the single atom study were used to decipher the structure of sub-nanometer clusters based on their HAADF-intensity. Such a micrograph is shown in Figure 2, where the morphology of the clusters are determined using single atom intensity as reference. Effect of tilting and channeling were not taken into account as they are insignificant for clusters less than 3 layers thick. For a successful application of this technique, atomic number of the elements needs to be sufficiently apart from each other. Accordingly, $\mathrm{Pd}$ single atoms can be differentiated from $\mathrm{Zr}(\mathrm{Z}=40)$ or lighter atoms and $\mathrm{Ba}$ $(Z=56)$ or heavier. Likewise, $P t$ single atoms can be differentiated from $\operatorname{Tm}(Z=69)$ or lighter and $\mathrm{Rn}$ $(\mathrm{Z}=86)$ or heavier. 
[1] Suenaga et al, Eur. Phys. J. Appl. Phys. (2011), 5433508

[2] Tizei et al, Ultramicroscopy (2016), 160 239-246

[3] O.L. Krivanek et al., Nature 464 (2010) 571-574

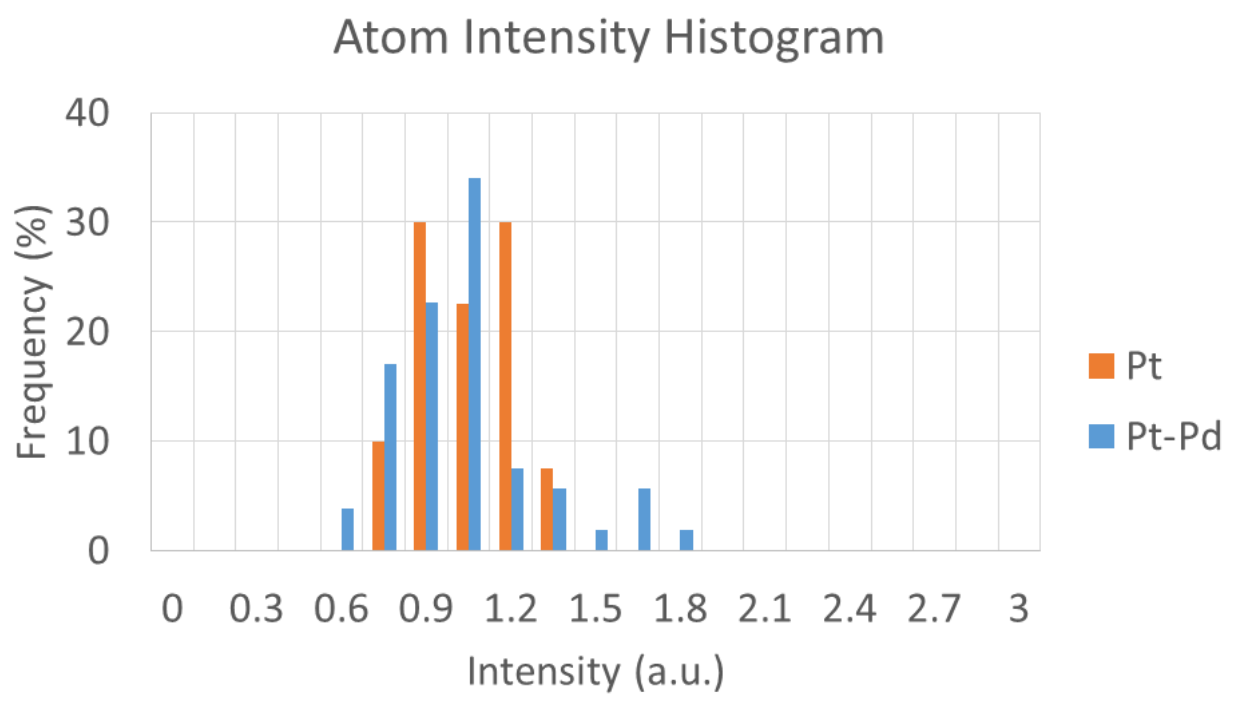

Figure 1. HAADF-STEM intensity histogram distribution of single atoms on the monometallic $\mathrm{Pt} / \mathrm{Al}_{2} \mathrm{O}_{3}$ catalyst and bimetallic $\mathrm{Pt}: \mathrm{Pd} / \mathrm{Al}_{2} \mathrm{O}_{3}$ catalyst
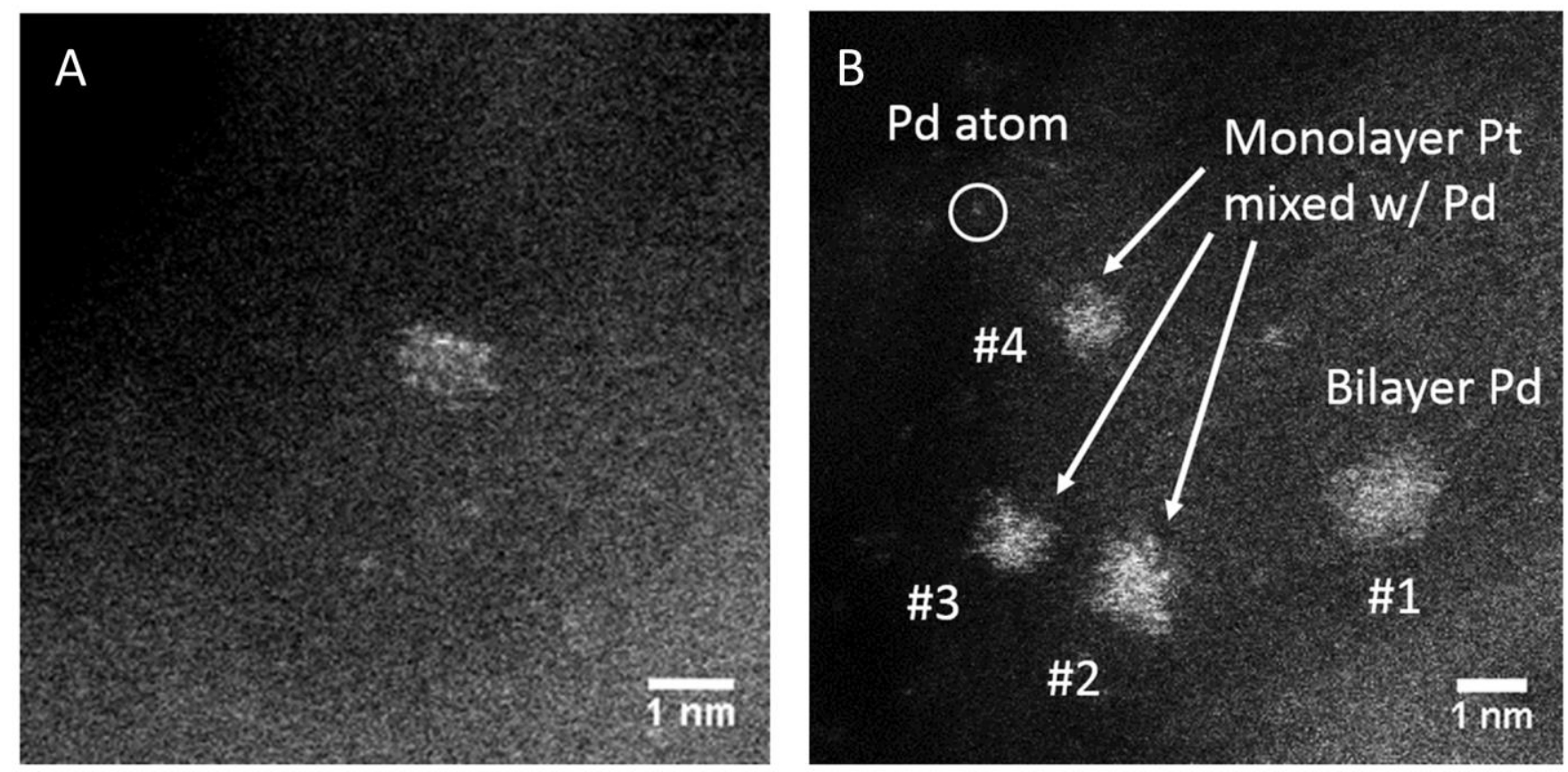

Figure 2. A) HAADF-STEM micrograph featuring a bimetallic nanoparticle on the $\mathrm{Pt}: \mathrm{Pd} / \mathrm{Al}_{2} \mathrm{O}_{3}$ catalyst B) HAADF-STEM micrographs of the $\mathrm{Pt}: \mathrm{Pd} / \mathrm{Al}_{2} \mathrm{O}_{3}$ catalyst featuring a bilayer Pd cluster (\#1) and three monolayer Pt clusters mixed with Pd atoms (\#2, \#3, \#4) 